Article

\title{
Impact of Small Molecules on Intermolecular G-Quadruplex Formation
}

\author{
Prabesh Gyawali ${ }^{1}$, Keshav GC ${ }^{2}$, Yue Ma ${ }^{3}{ }^{\circ}$, Sanjaya Abeysirigunawardena ${ }^{2}$, \\ Kazuo Nagasawa ${ }^{3}$ (D) and Hamza Balci ${ }^{1, *}$ \\ 1 Department of Physics, Kent State University, Kent, OH 44242, USA; pgyawal2@kent.edu \\ 2 Department of Chemistry and Biochemistry, Kent State University, Kent, OH 44242, USA; \\ kgc@kent.edu (K.G.); sabeysir@kent.edu (S.A.) \\ 3 Department of Biotechnology and Life Science, Tokyo University of Agriculture and Technology, Koganei, \\ Tokyo 184-8588, Japan; yue-ma@m2.tuat.ac.jp (Y.M.); knaga@cc.tuat.ac.jp (K.N.) \\ * Correspondence: hbalci@kent.edu; Tel.: +1-330-672-2577
}

Academic Editors: Liliya Yatsunyk and David Monchaud

Received: 9 February 2019; Accepted: 19 April 2019; Published: 20 April 2019

check for updates

\begin{abstract}
We performed single molecule studies to investigate the impact of several prominent small molecules (the oxazole telomestatin derivative L2H2-6OTD, pyridostatin, and Phen-DC 3 ) on intermolecular G-quadruplex (i-GQ) formation between two guanine-rich DNA strands that had 3-GGG repeats in one strand and 1-GGG repeat in the other (3+1 GGG), or 2-GGG repeats in each strand (2+2 GGG). Such structures are not only physiologically significant but have recently found use in various biotechnology applications, ranging from DNA-based wires to chemical sensors. Understanding the extent of stability imparted by small molecules on i-GQ structures, has implications for these applications. The small molecules resulted in different levels of enhancement in i-GQ formation, depending on the small molecule and arrangement of GGG repeats. The largest enhancement we observed was in the 3+1 GGG arrangement, where i-GQ formation increased by an order of magnitude, in the presence of L2H2-6OTD. On the other hand, the enhancement was limited to three-fold with Pyridostatin (PDS) or less for the other small molecules in the 2+2 GGG repeat case. By demonstrating detection of i-GQ formation at the single molecule level, our studies illustrate the feasibility to develop more sensitive sensors that could operate with limited quantities of materials.
\end{abstract}

Keywords: G-quadruplex; small molecule; single molecule; FRET; biosensor

\section{Introduction}

G-quadruplex structures (GQs) have emerged not only as promising targets for cancer therapy [1,2] or transcription-level [3-5] and translation-level [6] gene expression regulation, but they have also found use in various biotechnology applications [7]. As physiological relevance and technological potential of GQs became better established, research on identifying and synthesizing small molecules (SMs) that stabilize them, also experienced significant progress [8-11]. Prominence of GQ stabilizing SMs is typically characterized by their specificity to GQs, compared to double-stranded or single-stranded DNA (dsDNA or ssDNA), and by the increase these give rise to, in the thermal melting point $\left(\Delta \mathrm{T}_{\mathrm{m}}\right)$ of GQs. Pyridostatin (PDS), Phen-DC3, and oxazole telomestatin derivatives (OTD) are some of the prominent SMs in these respects (Figure 1A shows chemical structures of these SMs) [12-15]. In the particular context of human telomeric GQ (hGQ), $\Delta \mathrm{T}_{\mathrm{m}}=30{ }^{\circ} \mathrm{C}$ for Phen-DC $3[16], \Delta \mathrm{T}_{\mathrm{m}}=16{ }^{\circ} \mathrm{C}$ for L2H2-6OTD [17], and $\Delta \mathrm{T}_{\mathrm{m}}=35^{\circ} \mathrm{C}$ for PDS [18], have been reported under different ionic conditions (50-150 mM KCl). 
A

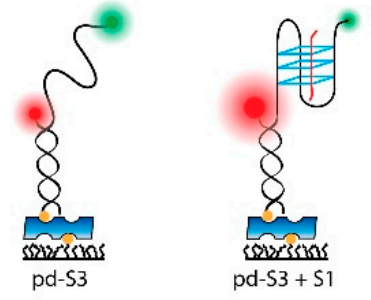

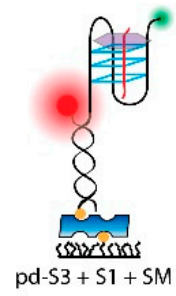

Cy 5 Biotin

Cу $3 \int \mathrm{S} 1$

\$86ris PEG

Neutravidin

Small Molecule (SM)

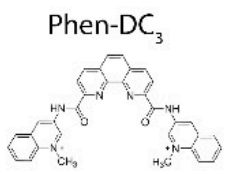

Pyridostatin

L2H2-6OTD
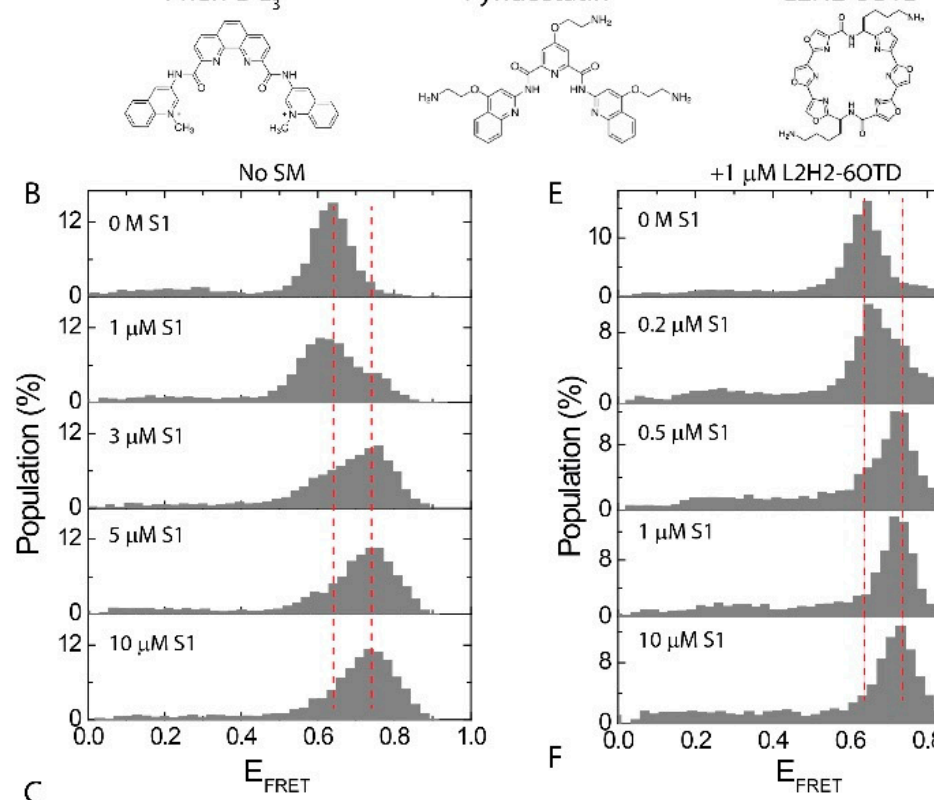

$\mathrm{E}$
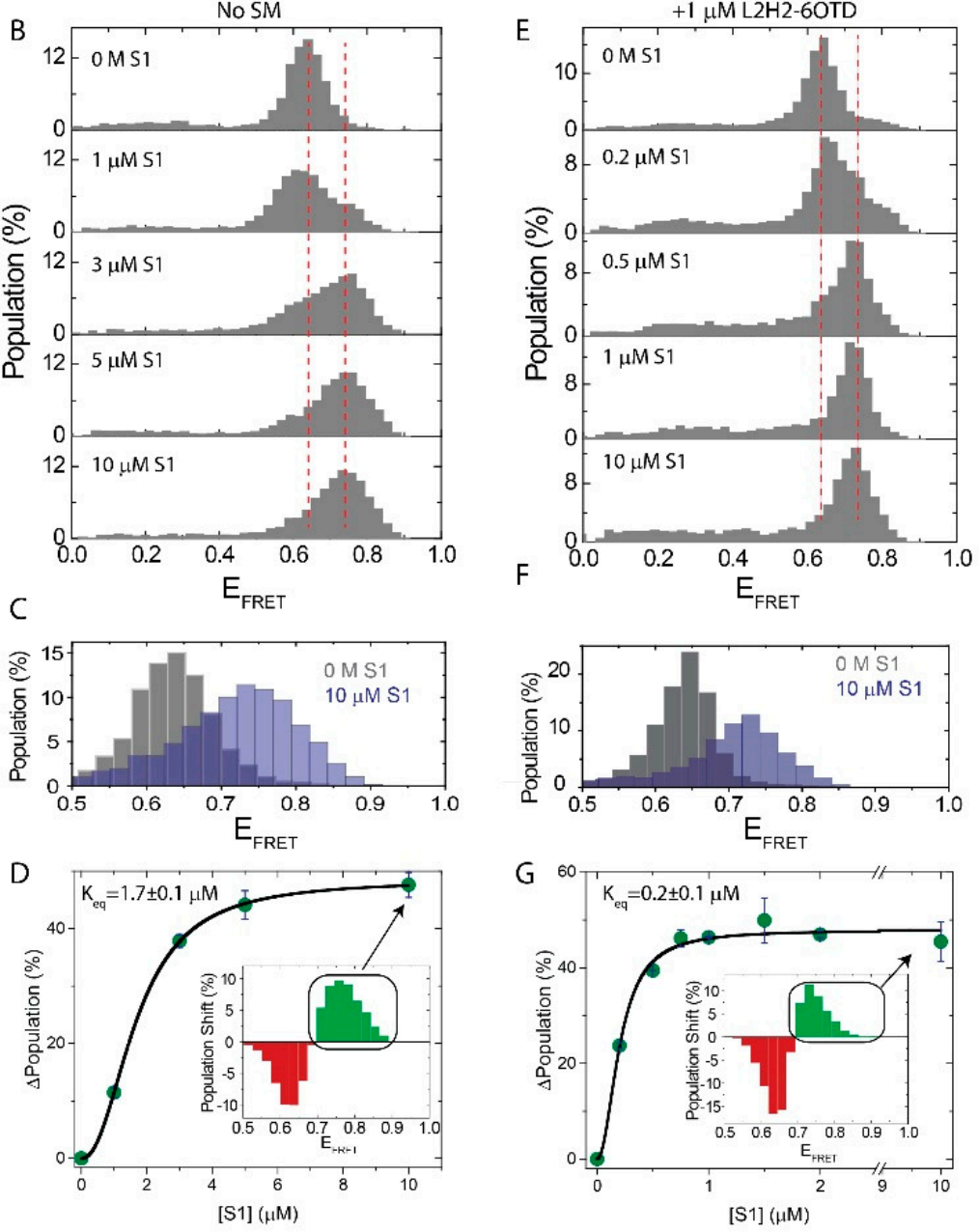

Figure 1. The effect of L2H2-6OTD on the i-GQ formation in 3+1 GGG configuration in the $150 \mathrm{mM} \mathrm{K}^{+}$. (A) Schematic of the DNA constructs and the smFRET assay. The bottom panel shows the chemical structures of the SMs used in the measurements. (B) S1 was titrated from $0 \mathrm{M}$ to $10 \mu \mathrm{M}$ in the absence of SM. Higher FRET states were populated as [S1] increased, suggesting i-GQ formation. The red dashed lines indicate the peak of the FRET distribution at $0 \mathrm{M}$ and $10 \mu \mathrm{M} \mathrm{S1}$. (C) $0 \mathrm{M}$ and $10 \mu \mathrm{M}$ S1 data were overlaid to illustrate the shift. (D) The transition to the i-GQ state was quantified by subtracting the FRET distribution at $0 \mathrm{M} \mathrm{S1}$ from that at corresponding [S1]. The total positive higher FRET population (green bars at the inset) was plotted as a function of [S1]. The black line was a Hill function fit. (E-G) were the equivalents of (B-D), respectively, when $1 \mu \mathrm{M} \mathrm{L2H2-6OTD} \mathrm{was} \mathrm{maintained}$ in the chamber, during S1 titration. $\mathrm{K}_{\mathrm{eq}}$ decreased from $1.7 \mu \mathrm{M}$ in the absence of SM to $0.2 \mu \mathrm{M}$ in the presence of $1 \mu \mathrm{M}$ L2H2-6OTD. 
Even though SMs are conventionally designed to stabilize intramolecular GQs, they may also facilitate intermolecular GQ (i-GQ) formation between G-rich neighboring regions of complementary strands. Bioinformatics studies showed that potential GQ-forming sequences (PQS), in which both strands of genomic DNA are involved in the GQ formation, co-localize with the functional sites in human genome and are more abundant than PQS within a single DNA strand [19]. The SMs that are designed to stabilize intramolecular GQs, could also promote and stabilize i-GQ between such strands, which may or may not be a desired effect, depending on the application. In addition, the concept of i-GQ formation by two or more strands of DNA has been used in various biotechnological applications, including G-wires [20,21], DNA-origami structures [22,23], and DNA-based devices, such as DNA walkers [24] and Heme-DNAzymes [25,26]. Biosensors that incorporate i-GQ structures, have been used to detect the presence of p53 or BRCA1 gene fragment $[27,28]$, or deletion of the LMP1 gene fragment [29,30], a metal ion [31], or a chemical [32,33]. In such applications, the two G-rich strands are brought to close-enough proximity to form a "split G-quadruplex" if one of the conditions listed above is satisfied. Whether or not an i-GQ is formed, can be detected via (i) a probe, such as an iridium compound that demonstrates enhanced luminescence upon binding to i-GQ [32]; (ii) the chemiluminescence signal that results from activation of the DNAzyme formed by i-GQ and hemin, in the presence of a metal ion or a chemical [31]; and (iii) a change in fluorescence signal, such as the quenching of fluorophores that are brought in close proximity upon i-GQ formation [28,34]. Since the performance of such a sensor depends on the efficiency of i-GQ formation, optimizing the sequences of the strands or providing SMs that facilitate i-GQ formation, would result in more sensitive sensors. There has been significant efforts for identifying and synthesizing GQ-stabilizing SMs [11,14,35-37], and improvements in their functionality, for different applications [38-41] where intramolecular GQs have generally been used as the scaffold. Whether such compounds function as efficiently in stabilizing $\mathrm{i}-\mathrm{GQ}$, is one of the questions we investigated in this study. Our particular focus was on detection, using single molecule methods, which provide unique capabilities and challenges, compared to ensemble level measurements. In particular, the enhanced sensitivity and low demand on materials are two promising aspects of using a single molecule scheme in sensor applications that involve i-GQ formation.

\section{Results and Discussion}

In order to study the impact of SMs on the i-GQ formation, we developed an smFRET assay where i-GQ formation is studied in the absence or presence of SMs. The DNA constructs and labeling positions were selected such that, formation of i-GQ between a surface-immobilized partial duplex DNA (pd-DNA) and a strand that is introduced into the chamber, results in a detectable increase in the FRET efficiency ( $\left.\mathrm{E}_{\mathrm{FRET}}\right)$. We studied two different configurations that involved telomeric repeats. In one case, three GGG repeats were in the surface-bound DNA (pd-S3) and another GGG-repeat was introduced as an additional strand (S1). This is called the 3+1 GGG configuration. In the other case, the surface-bound DNA (pd-S2) had two GGG-repeats and two additional GGG-repeats were introduced in another strand (S2). This is called the 2+2 GGG configuration. The 1+3 GGG configuration (monitoring FRET shift across pd-S1 while S3 is titrated) was not studied, since FRET efficiency would approach saturation for pd-S1, even due to the very short overhang ( $<10 \mathrm{nt})$, even before adding S3. Additionally, we did not explore any configuration that had more than three GGG repeats in one strand as that would result in the formation of an intramolecular GQ, which was not the focus of this study.

\subsection{Influence of $S M$ s on the $i-G Q$ Formation in $3+1$ GGG-Repeat Configuration}

Figure 1 shows smFRET measurements where pd-S3 is immobilized to the surface and $\mathrm{S} 1$ is titrated at $150 \mathrm{mM} \mathrm{K}^{+}$. In the absence of S1, pd-S3 shows a FRET distribution that is peaked at $\mathrm{E}_{\mathrm{FRET}}=0.63 \pm$ 0.05 , the uncertainty being the sigma value of the Gaussian fit. Adding increasing concentration of S1, results in the emergence and gradual population of a second peak at $\mathrm{E}_{\mathrm{FRET}}=0.75 \pm 0.06$. The dashed red lines marked these peak positions. To illustrate the change in the FRET distribution more clearly, 
the $0 \mathrm{M} \mathrm{S1}$ and $10 \mu \mathrm{M}$ S1 data were overlaid in Figure 1C. We interpreted this newly emerging high FRET population as an evidence for the i-GQ formation, which resulted in the compaction of pd-S3, in the form of a G-triplex (GT). In order to ensure that the observed increase in the FRET efficiency was due to the i-GQ formation, similar measurements were performed in $150 \mathrm{mM} \mathrm{LiCl}$ or $150 \mathrm{mM}$ TMAA, in which the i-GQ formation was not expected or was expected to be much weaker than that in the $150 \mathrm{mM} \mathrm{K}^{+}$. In both cases the FRET peak did not shift to higher values, even at $10 \mu \mathrm{M}$ S1 (Figure 2A), suggesting that the compaction we observed in $\mathrm{K}^{+}$was due to the i-GQ formation. To quantify how the fraction of this high FRET population changed with S1 concentration ([S1]), we subtracted the FRET distribution at $0 \mathrm{M} \mathrm{S1}$, from the FRET distributions at the respective [S1]. As both FRET distributions were normalized to have a total area of $100 \%$, the resulting subtraction histogram had a net zero area, with the negative areas representing depleted states and positive areas representing newly emerging states, upon adding S1. An example of the subtraction histogram is shown in the inset of Figure 1D. Integrating the green bins in this subtraction histogram, resulted in the total percentage of newly emerging states, which we interpreted as a measure of the i-GQ population. By plotting this positive area as a function of [S1] and performing a Hill function fit, an equilibrium constant could be obtained. Figure 1D shows the results of this analysis, with $\mathrm{K}_{\mathrm{eq}}=1.7 \pm 0.1 \mu \mathrm{M}$. An alternative method to analyze this type of data was to fit the FRET histograms to a function with two Gaussian peaks and monitor how these populations varied with [S1]. However, the close proximity of the peaks, $\mathrm{E}_{\mathrm{FRET}}=0.63 \pm 0.05$ and $\mathrm{E}_{\mathrm{FRET}}=0.75 \pm 0.06$, made this approach prone to fitting errors.
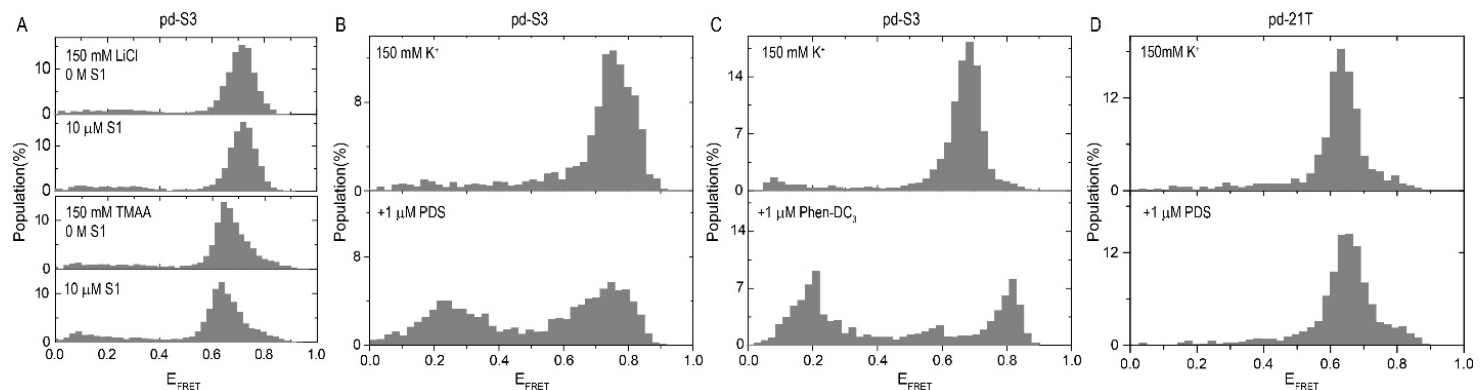

Figure 2. (A) Even in the presence of $10 \mu \mathrm{M}$ S1, no shift in the FRET was observed for the pd-S3 construct in the $150 \mathrm{mM} \mathrm{LiCl}$ (top) or $150 \mathrm{mM}$ TMAA (bottom), suggesting the shift in $\mathrm{KCl}$ to be due to the i-GQ formation. (B) Demonstration of the change in the FRET histogram upon adding $1 \mu \mathrm{M}$ PDS. A significant low FRET population emerged which was considered to be due to the interaction of PDS with the fluorophores. (C) Similar low FRET states were observed when $1 \mu \mathrm{M}$ Phen-DC 3 was introduced. (D) Such low FRET states were not observed when PDS was introduced in a chamber that contained pd-21T construct, an unstructured DNA (polythymidine) of similar length to pd-S3.

In order to test whether the SMs had any effect on this type of i-GQ formation, we performed the same S1 titration in the presence of SM. We used an oxazole telomestatin derivative (L2H2-6OTD), PDS, and Phen-DC3 as model SMs, at $1 \mu \mathrm{M}$ concentration. Among these SMs, only the L2H2-6OTD demonstrated significant improvement in the GQ formation and stability, while the other two SMs had unexpected effects on the smFRET distributions that prevented reliably quantifying their impact. The data on the L2H2-6OTD have been presented before the PDS and the Phen-DC 3 have been discussed. Figure 1E shows S1 titration in the presence of $1 \mu \mathrm{M}$ L2H2-6OTD, Figure 1F shows the overlay of $0 \mathrm{M}$ and $10 \mu \mathrm{M}$ S1 data, and Figure 1G shows the results of the subtraction analysis, where $\mathrm{K}_{\mathrm{eq}}=0.2 \pm 0.1 \mu \mathrm{M}$ was obtained. This $\mathrm{K}_{\mathrm{eq}}$ is an order of magnitude smaller than that observed in the absence of SMs, suggesting a significant enhancement in the i-GQ formation in the presence of L2H2-6OTD. Similar measurements were also performed, using electrophoretic mobility shift assay (EMSA), where the S3 (GGGTTAGGGTTAGGG) strand was radiolabeled with phosphorus-32 (P32). Titrating S1 strand while keeping the S3 concentration at $10 \mathrm{nM}$, resulted in the emergence of a new band. These data are shown in Figure S1 in the Supplementary Materials. 
Unlike L2H2-6OTD, the impact of Phen-DC ${ }_{3}$ and PDS on the i-GQ formation in the 3+1 GGG configuration, could not be quantified. Upon adding these SMs (before adding S1), either a new low FRET peak or a broad distribution of low FRET states emerged (Figure 2B,C). The reason behind these low FRET states was not clear. Quenching of the acceptor fluorophore by the SM was one of the possibilities, as it was demonstrated that PDS and Phen-DC 3 had such an effect when they bound to the close proximity of Cy5 [15]. As these low FRET states were observed before S1 was added, PDS and Phen-DC $\mathrm{D}_{3}$ would have needed to interact with the intermediate folding states, such as G-triplex. Such low FRET states were not observed when the PDS was introduced in a chamber that contained a DNA construct with an unstructured overhang (pd-21T), of similar length, to that of the pd-S3 construct (Figure 2D).

\subsection{Influence of SMs on the $i-G Q$ Formation in the $2+2$ GGG-Repeat Configuration}

We also tested the impact of SMs on the i-GQ formation between pd-S2 and S2 (see Table 1 for sequences and Figure 3A for a schematic of the assay), using EMSA (Supplementary Materials Figure S1) and smFRET. At $150 \mathrm{mM} \mathrm{K}^{+}$, introducing higher concentrations of S2 resulted in the compaction of the structure in pd-S2, as demonstrated by a shift to higher FRET values in Figure 3B. In the absence of S2, pd-S2 demonstrated a peak at $\mathrm{E}_{\mathrm{FRET}}=0.78 \pm 0.04$. Introducing $\mathrm{S} 2$ resulted in the emergence and gradual population of a higher FRET peak at $\mathrm{E}_{\mathrm{FRET}}=0.87 \pm 0.02$. Figure $3 \mathrm{C}$ shows the histograms at $0 \mathrm{M}$ and $10 \mu \mathrm{M} \mathrm{S2}$, which demonstrated this shift. We interpreted this compaction to be due to the formation of the i-GQ between the pd-S2 and S2. Figure 3D shows the results of the subtraction analysis where the FRET distribution at $0 \mathrm{MS} 2$ was subtracted from that at the respective [S2]. A Hill function fit resulted in $K_{\mathrm{eq}}=2.3 \pm 0.5 \mu \mathrm{M}$. We then repeated these measurements in the presence of $1 \mu \mathrm{M}$ L2H2-6OTD, PDS or Phen-DC 3 , while all other ingredients of the imaging buffer were kept identical (Figure 3E-M). Figure 3F,I,L show the shift in the FRET histograms at $0 \mathrm{M}$ and $10 \mu \mathrm{M}$ S2 for L2H2-6OTD, PDS, and Phen-DC3, respectively. Similar subtraction analysis and Hill function fit the result in $\mathrm{K}_{\mathrm{eq}}=2.1 \pm 0.2 \mu \mathrm{M}$ for $\mathrm{L} 2 \mathrm{H} 2-6 \mathrm{OTD}$ (Figure $3 \mathrm{G}$ ), $\mathrm{K}_{\mathrm{eq}}=0.8 \pm 0.3 \mu \mathrm{M}$ for PDS (Figure 3J), and $K_{\text {eq }}=2.2 \pm 0.4 \mu \mathrm{M}$ for Phen-DC3 (Figure 3M). Unlike the L2H2-6OTD and Phen-DC3, which did not result in a significant enhancement of the i-GQ formation in the 2+2 GGG configuration, the PDS resulted in a three-fold enhancement.

Overall, these results suggested that these SMs either did not facilitate the i-GQ formation in the 2+2 GGG configuration or had a relatively weaker impact, compared to the order of magnitude enhancement observed in the 3+1 GGG configuration. It is particularly interesting that L2H2-6OTD enhanced the i-GQ formation in the 3+1 GGG configuration, by an order of magnitude, but essentially had no impact on the i-GQ formation in the $2+2$ GGG configuration. The reasons behind this very interesting and potentially significant observation was not clear, but there were several different possibilities. It was possible that the folding conformation attained in the 3+1 GGG configuration was more favorable to the L2H2-OTD stacking, compared to the conformation in the 2+2 GGG configuration. Another possibility was the higher stability of the i-GQ structure formed in the $3+1$ GGG configuration, which resulted in smaller fluctuations in the structure and allowed stacking of the L2H2-6OTD. This might have been particularly important for the transiently folded i-GQ structures, which might have been stable enough to be bound and further stabilized by the L2H2-6OTD in the case of the 3+1 GGG but not in the case of 2+2 GGG. Finally, it is possible that L2H2-6OTD facilitated the transition to a fully folded i-GQ from certain intermediate folding states that were more likely to form in the 3+1 GGG configuration, compared to the 2+2 GGG configuration, such as the G-triplex. It is likely that different scenarios were prevalent for different SMs, based on their structural details, where on the i-GQ structure, they bound their affinity to the intermediate folding states and their tolerance to the fluctuations in the structure. A conclusive answer to this interesting question would require both structural studies and dynamic studies with a high enough time resolution to probe the i-GQ folding process. 
Table 1. DNA sequences used for this study. The sequences that formed the stem are shown in bold fonts. Stem is complementary to an 18nt segment in the pd-S3, pd-S2, and pd-21T.

\begin{tabular}{cc}
\hline Construct & Sequence $\mathbf{( 5}^{\prime}-\mathbf{3}^{\prime} \mathbf{)}$ \\
\hline Stem & Cy5-GCCTCGCTGCCGTCGCCA-Biotin \\
pd-S3 & TGGCGACGGCAGCGAGG AGGGTTAGGGTTAGGGTTAG-Cy3 + Stem \\
pd-S2 & TGG CGA CGG CAG CGA GGC AGGGTTAGGGTTAG-Cy3 + Stem \\
pd-21T & TGG CGA CGG CAG CGA GGC TTTTTTTTTTTTTTTTTTTT-Cy3 + Stem \\
S1 & AGGGT \\
S2 & GGGTTAGGG \\
\hline
\end{tabular}

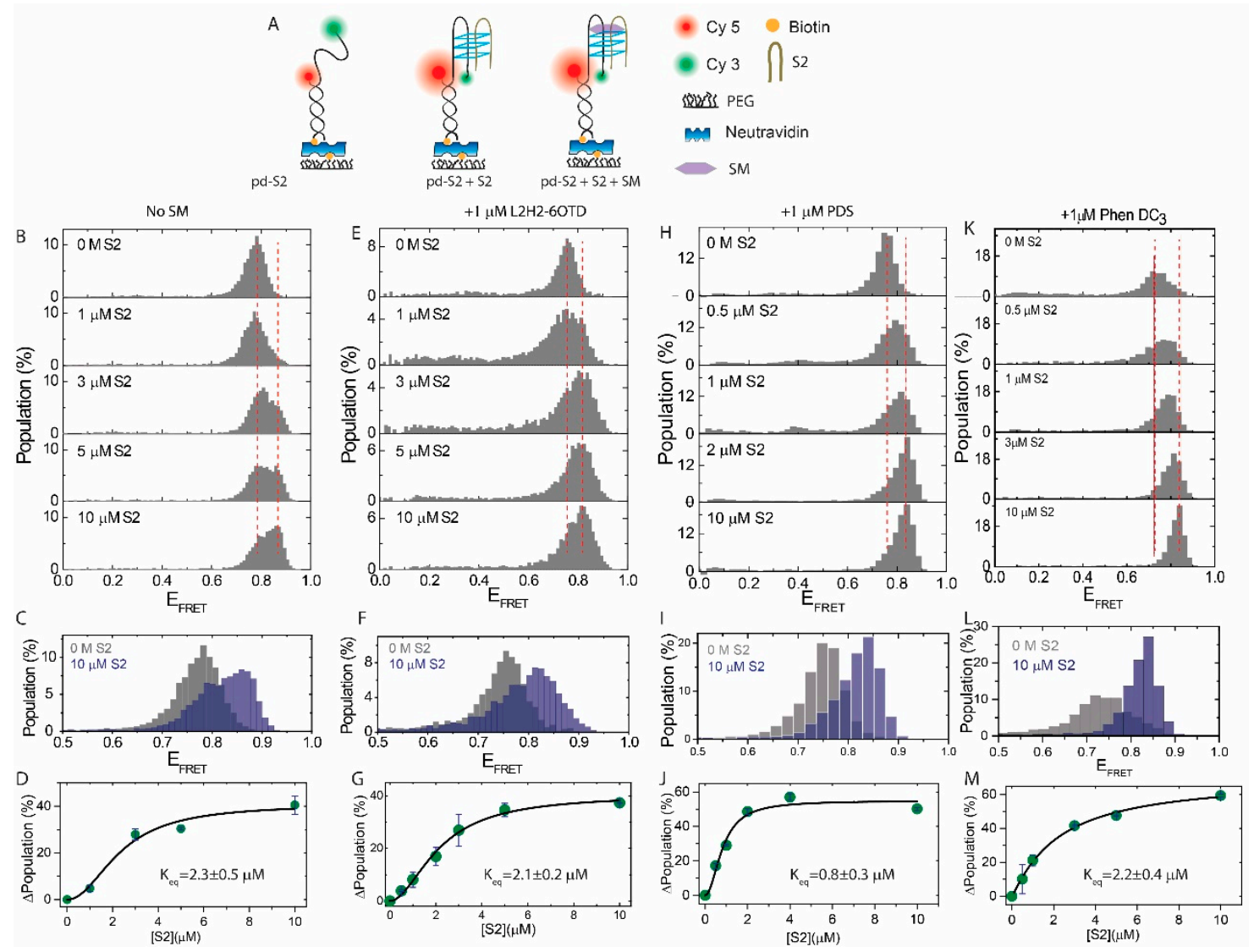

Figure 3. The effect of SMs on i-GQ formation in 2+2 GGG-repeat configuration in $150 \mathrm{mM} \mathrm{K}^{+}$. (A) Schematic of the DNA constructs and the smFRET assay. (B) S2 was titrated from $0 \mathrm{M}$ to $10 \mu \mathrm{M}$ in the absence of SM. Higher FRET states were populated in the FRET histogram as [S2] increased, suggesting i-GQ formation. The two red dashed lines indicated the peak of the FRET distribution at $0 \mathrm{M}$ and $10 \mu \mathrm{M}$ S2. (C) $0 \mathrm{M}$ and $10 \mu \mathrm{M}$ S2 data were overlaid to illustrate the shift. (D) Formation of i-GQ state was quantified by subtracting the FRET distribution at $0 \mathrm{M} \mathrm{S2}$, from that at the corresponding [S2]. The cumulative of positive higher FRET population was plotted as a function of [S2]. The black line was a Hill function fit. (E-G) were identical to (B-D), respectively, except $1 \mu$ M L2H2-6OTD was maintained in the chamber during $\mathrm{S} 2$ titration. $(\mathbf{H}-\mathbf{J})$ showed the same measurements in the presence of $1 \mu \mathrm{M}$ PDS, and $(\mathbf{K}-\mathbf{M})$ in the presence of $1 \mu \mathrm{M}$ Phen-DC ${ }_{3}$.

\section{Materials and Methods}

Prism-type total internal reflection fluorescence microscopy measurements were performed on an Olympus IX-71 microscope, equipped with an Olympus 60x, 1.20 NA water objective, and an Andor Ixon EMCCD camera (iXon DV 887-BI EMCCD, Andor Technology, South Windsor, CT, USA). A $\lambda=532 \mathrm{~nm}$ green laser (SpectraPhysics Excelsior) was used as the excitation source. A total of $1 \%$ of PEG molecules were tagged with biotin to provide attachment points for the biotinylated 
DNA molecules, which bind to the biotin-PEG via neutravidin. To provide adequate statistics for the analysis, 250-350 DNA molecules per imaging area $\left(\sim 5 \times 10^{3} \mu \mathrm{m}^{2}\right)$ were targeted as the ideal density in most measurements. The imaging buffer contained Tris base (50 mM, pH 7.5), $2 \mathrm{mM}$ Trolox, $0.8 \mathrm{mg} / \mathrm{mL}$ glucose, $0.1 \mathrm{mg} / \mathrm{mL}$ bovine serum albumin (BSA), $0.1 \mathrm{mg} / \mathrm{mL}$ glucose oxidase, $0.02 \mathrm{mg} / \mathrm{mL}$ catalase, $150 \mathrm{mM} \mathrm{KCl}$ and $2 \mathrm{mM} \mathrm{MgCl}_{2}$. $\mathrm{LiCl}$ or TMAA [42] were used, instead of $\mathrm{KCl}$ in some control measurements. A total of $1 \mu \mathrm{M} \mathrm{SM}$ and varying concentrations of the second DNA strand (S1 or S2) were also added to the imaging buffer, in relevant measurements. Recording of the data started after incubating all components in the chamber, for 15 minutes. Short movies (15 frames) or long movies (1500-3000 frames) were recorded at a frame integration time of $100 \mathrm{msec}$. Each of the histograms in Figures 1-3 was constructed from single molecule data, obtained from 20-30 different movies, each imaging a different site on the surface, and represented the distribution of the FRET efficiency for several thousand molecules.

Both, the fluorescently-labeled and the unlabeled DNA oligonucleotides were purchased as PAGE or HPLC, purified from the Integrated DNA Technologies (IDT). In order to make the pdDNA constructs, the Stem strand (Table 1) was annealed with the relevant strand at $90^{\circ} \mathrm{C}$, for $3 \mathrm{~min}$, followed by a slow cooling to room temperature, over $2-3 \mathrm{~h}$.

\section{Conclusions}

This study demonstrated the feasibility, sensitivity, and material demands of detecting the i-GQ formation, using a single molecule platform in the presence or absence of GQ stabilizing SMs. It presented the range of impact that could be expected when SMs were utilized to enhance the i-GQ formation for different configurations of GGG repeats. An order of magnitude enhancement in the i-GQ formation was observed in 3+1 GGG configuration with the L2H2-6OTD. The 2+2 GGG configuration was less efficient at the i-GQ formation, with or without SMs. The largest enhancement in this configuration was three-fold with PDS. This was reasonable, as it would be easier for intermediate folding conformations (predecessors of the i-GQ) to form, when three GGG repeats were in one strand. In a physiological setting, these results could be desired for some applications, e.g., when elevated synthetic lethality and genomic instability in cancer cells was desired. However, in applications where specific targeting of a particular GQ was desired, e.g., to regulate transcription level gene expression, then these SMs might have given rise to the i-GQ formation at sites where they would otherwise not form. Our study also had consequences for biotechnology applications that utilized and depended on the i-GQ formation. We demonstrated that with the proper design of DNA constructs and the right SM, it was possible to enhance the i-GQ formation, hence, the signal of a sensor could also be enhanced by an order of magnitude. Finally, fluorescence-based detection schemes needed to take into account the potential photophysical effects that might arise due to SM-dye and dye-GQ interactions [43]. While the L2H2-6OTD did not have these limitations, both Phen-DC3 and PDS gave rise to complications. Therefore, if these molecules were to be used for sensor applications, either possible quenching of the fluorophores should be well-characterized or non-fluorescence-based methods should be used for detection.

Supplementary Materials: The supplementary materials are available online.

Author Contributions: Conceptualization, H.B.; data curation, methodology, and formal analysis P.G., K.G., and H.B.; writing-original draft preparation, H.B.; writing-review and editing, P.G., K.G., S.A., Y.M., K.N., and H.B.

Funding: This research was funded by National Institutes of Health [1R15GM109386 and 1R15GM123443 to H.B.].

Conflicts of Interest: The authors declare no conflict of interest. 


\section{References}

1. McLuckie, K.I.; Di Antonio, M.; Zecchini, H.; Xian, J.; Caldas, C.; Krippendorff, B.F.; Tannahill, D.; Lowe, C.; Balasubramanian, S. G-quadruplex DNA as a molecular target for induced synthetic lethality in cancer cells. J. Am. Chem. Soc. 2013, 135, 9640-9643. [CrossRef] [PubMed]

2. Merle, P.; Gueugneau, M.; Teulade-Fichou, M.P.; Muller-Barthelemy, M.; Amiard, S.; Chautard, E.; Guetta, C.; Dedieu, V.; Communal, Y.; Mergny, J.L.; et al. Highly efficient radiosensitization of human glioblastoma and lung cancer cells by a g-quadruplex DNA binding compound. Sci. Rep. 2015, 5, 16255. [CrossRef] [PubMed]

3. Fernando, H.; Reszka, A.P.; Huppert, J.; Ladame, S.; Rankin, S.; Venkitaraman, A.R.; Neidle, S.; Balasubramanian, S. A conserved quadruplex motif located in a transcription activation site of the human c-kit oncogene. Biochemistry 2006, 45, 7854-7860. [CrossRef]

4. Fernando, H.; Sewitz, S.; Darot, J.; Tavare, S.; Huppert, J.L.; Balasubramanian, S. Genome-wide analysis of a g-quadruplex-specific single-chain antibody that regulates gene expression. Nucleic Acids Res. 2009, 37, 6716-6722. [CrossRef]

5. Mitchell, T.; Ramos-Montoya, A.; Di Antonio, M.; Murat, P.; Ohnmacht, S.; Micco, M.; Jurmeister, S.; Fryer, L.; Balasubramanian, S.; Neidle, S.; et al. Downregulation of androgen receptor transcription by promoter g-quadruplex stabilization as a potential alternative treatment for castrate-resistant prostate cancer. Biochemistry 2013, 52, 1429-1436. [CrossRef]

6. Morris, M.J.; Negishi, Y.; Pazsint, C.; Schonhoft, J.D.; Basu, S. An rna g-quadruplex is essential for cap-independent translation initiation in human vegf ires. J. Am. Chem. Soc. 2010, 132, 17831-17839. [CrossRef]

7. Mergny, J.L.; Sen, D. DNA quadruple helices in nanotechnology. Chem. Rev. 2019. [CrossRef]

8. Nasiri, H.R.; Bell, N.M.; McLuckie, K.I.; Husby, J.; Abell, C.; Neidle, S.; Balasubramanian, S. Targeting a c-myc g-quadruplex DNA with a fragment library. Chem. Commun. (Camb.) 2014, 50, 1704-1707. [CrossRef]

9. Neidle, S. A personal history of quadruplex-small molecule targeting. Chem. Rec. 2015. [CrossRef]

10. Ohnmacht, S.A.; Varavipour, E.; Nanjunda, R.; Pazitna, I.; Di Vita, G.; Gunaratnam, M.; Kumar, A.; Ismail, M.A.; Boykin, D.W.; Wilson, W.D.; et al. Discovery of new g-quadruplex binding chemotypes. Chem. Commun. (Camb.) 2014, 50, 960-963. [CrossRef] [PubMed]

11. Rahman, K.M.; Tizkova, K.; Reszka, A.P.; Neidle, S.; Thurston, D.E. Identification of novel telomeric g-quadruplex-targeting chemical scaffolds through screening of three nci libraries. Bioorg. Med. Chem. Lett. 2012, 22, 3006-3010. [CrossRef] [PubMed]

12. Chung, W.J.; Heddi, B.; Hamon, F.; Teulade-Fichou, M.P.; Phan, A.T. Solution structure of a g-quadruplex bound to the bisquinolinium compound phen-dc(3). Angew Chem. Int. Ed. Engl. 2014, 53, 999-1002. [CrossRef]

13. De Rache, A.; Mergny, J.L. Assessment of selectivity of g-quadruplex ligands via an optimised fret melting assay. Biochimie 2015, 115, 194-202. [CrossRef]

14. Iida, K.; Nagasawa, K. Macrocyclic polyoxazoles as g-quadruplex ligands. Chem. Rec. 2013, 13, 539-548. [CrossRef] [PubMed]

15. Le, D.D.; Di Antonio, M.; Chan, L.K.; Balasubramanian, S. G-quadruplex ligands exhibit differential g-tetrad selectivity. Chem. Commun. (Camb.) 2015, 51, 8048-8050. [CrossRef] [PubMed]

16. De Cian, A.; Delemos, E.; Mergny, J.L.; Teulade-Fichou, M.P.; Monchaud, D. Highly efficient g-quadruplex recognition by bisquinolinium compounds. J. Am. Chem. Soc. 2007, 129, 1856-1857. [CrossRef] [PubMed]

17. Iida, K.; Majima, S.; Nakamura, T.; Seimiya, H.; Nagasawa, K. Evaluation of the interaction between long telomeric DNA and macrocyclic hexaoxazole (6otd) dimer of a g-quadruplex ligand. Molecules 2013, 18, 4328-4341. [CrossRef]

18. Rodriguez, R.; Muller, S.; Yeoman, J.A.; Trentesaux, C.; Riou, J.F.; Balasubramanian, S. A novel small molecule that alters shelterin integrity and triggers a DNA-damage response at telomeres. J. Am. Chem. Soc. 2008, 130, 15758-15759. [CrossRef]

19. Kudlicki, A.S. G-quadruplexes involving both strands of genomic DNA are highly abundant and colocalize with functional sites in the human genome. PLOS ONE 2016, 11, e0146174. [CrossRef]

20. Bose, K.; Lech, C.J.; Heddi, B.; Phan, A.T. High-resolution afm structure of DNA g-wires in aqueous solution. Nat. Commun. 2018, 9, 1959. [CrossRef] 
21. Marsh, T.C.; Henderson, E. G-wires: Self-assembly of a telomeric oligonucleotide, d(ggggttgggg), into large superstructures. Biochemistry 1994, 33, 10718-10724. [CrossRef]

22. Endo, M.; Sugiyama, H. DNA origami nanomachines. Molecules 2018, 23, 1766. [CrossRef]

23. Rajendran, A.; Endo, M.; Hidaka, K.; Tran, P.L.; Mergny, J.L.; Sugiyama, H. Controlling the stoichiometry and strand polarity of a tetramolecular g-quadruplex structure by using a DNA origami frame. Nucleic Acids Res. 2013, 41, 8738-8747. [CrossRef] [PubMed]

24. Yeo, Q.Y.; Loh, I.Y.; Tee, S.R.; Chiang, Y.H.; Cheng, J.; Liu, M.H.; Wang, Z.S. A DNA bipedal nanowalker with a piston-like expulsion stroke. Nanoscale 2017, 9, 12142-12149. [CrossRef]

25. Kosman, J.; Juskowiak, B. Peroxidase-mimicking dnazymes for biosensing applications: A review. Anal. Chim. Acta 2011, 707, 7-17. [CrossRef] [PubMed]

26. Sen, D.; Poon, L.C. Rna and DNA complexes with hemin [fe(iii) heme] are efficient peroxidases and peroxygenases: How do they do it and what does it mean? Crit. Rev. Biochem. Mol. Biol. 2011, 46, 478-492. [CrossRef]

27. Shimron, S.; Wang, F.; Orbach, R.; Willner, I. Amplified detection of DNA through the enzyme-free autonomous assembly of hemin/g-quadruplex dnazyme nanowires. Anal. Chem. 2012, 84, 1042-1048. [CrossRef] [PubMed]

28. Zhou, H.; Wu, Z.S.; Shen, G.L.; Yu, R.Q. Intermolecular g-quadruplex structure-based fluorescent DNA detection system. Biosens. Bioelectron. 2013, 41, 262-267. [CrossRef]

29. He, H.Z.; Chan, D.S.; Leung, C.H.; Ma, D.L. A highly selective g-quadruplex-based luminescent switch-on probe for the detection of gene deletion. Chem. Commun. (Camb.) 2012, 48, 9462-9464. [CrossRef] [PubMed]

30. Wang, M.; He, B.; Lu, L.; Leung, C.H.; Mergny, J.L.; Ma, D.L. Label-free luminescent detection of lmp1 gene deletion using an intermolecular g-quadruplex-based switch-on probe. Biosens. Bioelectron. 2015, 70, 338-344. [CrossRef]

31. Freeman, R.; Liu, X.; Willner, I. Chemiluminescent and chemiluminescence resonance energy transfer (cret) detection of DNA, metal ions, and aptamer-substrate complexes using hemin/g-quadruplexes and cdse/zns quantum dots. J. Am. Chem. Soc. 2011, 133, 11597-11604. [CrossRef]

32. Ma, D.L.; Wang, M.; He, B.; Yang, C.; Wang, W.; Leung, C.H. A luminescent cocaine detection platform using a split g-quadruplex-selective iridium(iii) complex and a three-way DNA junction architecture. ACS Appl. Mater. Interfaces 2015, 7, 19060-19067. [CrossRef] [PubMed]

33. Nasiri, A.H.; Wurm, J.P.; Immer, C.; Weickhmann, A.K.; Wohnert, J. An intermolecular g-quadruplex as the basis for gtp recognition in the class v-gtp aptamer. RNA 2016, 22, 1750-1759. [CrossRef]

34. Merkina, E.E.; Fox, K.R. Kinetic stability of intermolecular DNA quadruplexes. Biophys. J. 2005, 89, 365-373. [CrossRef] [PubMed]

35. Diveshkumar, K.V.; Sakrikar, S.; Harikrishna, S.; Dhamodharan, V.; Pradeepkumar, P.I. Targeting promoter g-quadruplex dnas by indenopyrimidine-based ligands. ChemMedChem 2014, 9, 2754-2765. [CrossRef] [PubMed]

36. Muller, S.; Laxmi-Reddy, K.; Jena, P.V.; Baptiste, B.; Dong, Z.; Godde, F.; Ha, T.; Rodriguez, R.; Balasubramanian, S.; Huc, I. Targeting DNA g-quadruplexes with helical small molecules. Chembiochem 2014, 15, 2563-2570. [CrossRef] [PubMed]

37. Ohnmacht, S.A.; Neidle, S. Small-molecule quadruplex-targeted drug discovery. Bioorg. Med. Chem. Lett. 2014, 24, 2602-2612. [CrossRef] [PubMed]

38. Iida, K.; Nakamura, T.; Yoshida, W.; Tera, M.; Nakabayashi, K.; Hata, K.; Ikebukuro, K.; Nagasawa, K. Fluorescent-ligand-mediated screening of g-quadruplex structures using a DNA microarray. Angew Chem. Int. Ed. Engl. 2013, 52, 12052-12055. [CrossRef]

39. Largy, E.; Granzhan, A.; Hamon, F.; Verga, D.; Teulade-Fichou, M.P. Visualizing the quadruplex: From fluorescent ligands to light-up probes. Top. Curr. Chem. 2013, 330, 111-177. [PubMed]

40. Verga, D.; Hamon, F.; Poyer, F.; Bombard, S.; Teulade-Fichou, M.P. Photo-cross-linking probes for trapping g-quadruplex DNA. Angew Chem. Int. Ed. Engl. 2014, 53, 994-998. [CrossRef] [PubMed]

41. Iida, K.; Tera, M.; Hirokawa, T.; Shin-ya, K.; Nagasawa, K. G-quadruplex recognition by macrocyclic hexaoxazole (6otd) dimer: Greater selectivity than monomer. Chem. Commun. (Camb.) 2009, 6481-6483. [CrossRef] [PubMed] 
42. Marchand, A.; Gabelica, V. Native electrospray mass spectrometry of DNA g-quadruplexes in potassium solution. J. Am. Soc. Mass. Spectrom. 2014, 25, 1146-1154. [CrossRef] [PubMed]

43. Sondergaard, S.; Aznauryan, M.; Haustrup, E.K.; Schiott, B.; Birkedal, V.; Corry, B. Dynamics of fluorescent dyes attached to g-quadruplex DNA and their effect on fret experiments. Chemphyschem 2015. [CrossRef] [PubMed]

Sample Availability: Samples of the compounds are available either commercially (PDS and Phen-DC 3 ) or (L2H2-OTD) from the authors.

(c)

(C) 2019 by the authors. Licensee MDPI, Basel, Switzerland. This article is an open access article distributed under the terms and conditions of the Creative Commons Attribution (CC BY) license (http://creativecommons.org/licenses/by/4.0/). 